Article

\title{
Stabilization Effects of Dichotomous Noise on the Lifetime of the Superconducting State in a Long Josephson Junction
}

\author{
Claudio Guarcello ${ }^{1,2, *}$, Davide Valenti ${ }^{1}$, Angelo Carollo ${ }^{1}$ and Bernardo Spagnolo ${ }^{1,2,3}$ \\ ${ }^{1}$ Dipartimento di Fisica e Chimica, Interdisciplinary Theoretical Physics Group, \\ Università di Palermo and CNISM, Unità di Palermo, Viale delle Scienze, Edificio 18, \\ I-90128 Palermo, Italy; E-Mails: davide.valenti@unipa.it (D.V.); angelo.carollo@ unipa.it (A.C.); \\ bernardo.spagnolo@unipa.it (B.S.) \\ ${ }^{2}$ Radiophysics Department, Lobachevsky State University of Nizhni Novgorod, 23 Gagarin Avenue, \\ Nizhni Novgorod 603950, Russia \\ ${ }^{3}$ Istituto Nazionale di Fisica Nucleare, Sezione di Catania, Via S. Sofia 64, I-90123 Catania, Italy \\ * Author to whom correspondence should be addressed; E-Mail: claudio.guarcello@unipa.it.
}

Academic Editors: Demosthenes Ellinas, Giorgio Kaniadakis, Jiannis Pachos and Antonio M. Scarfone Received: 6 March 2015 / Accepted: 30 April 2015 / Published: 6 May 2015

\begin{abstract}
We investigate the superconducting lifetime of a long overdamped current-biased Josephson junction, in the presence of telegraph noise sources. The analysis is performed by randomly choosing the initial condition for the noise source. However, in order to investigate how the initial value of the dichotomous noise affects the phase dynamics, we extend our analysis using two different fixed initial values for the source of random fluctuations. In our study, the phase dynamics of the Josephson junction is analyzed as a function of the noise signal intensity, for different values of the parameters of the system and external driving currents. We find that the mean lifetime of the superconductive metastable state as a function of the noise intensity is characterized by nonmonotonic behavior, strongly related to the soliton dynamics during the switching towards the resistive state. The role of the correlation time of the noise source is also taken into account. Noise-enhanced stability is observed in the investigated system.
\end{abstract}

Keywords: long Josephson junction; metastability; dichotomous noise; mean switching time; nonlinear relaxation time; noise enhanced stability; nonequilibrium statistical mechanics 


\section{Introduction}

Often, in solid state physics, the electric resistance of real systems shows fluctuations related to defects that modify the electric properties of the conductors. The influence of these defects therefore causes random modifications in the electric resistance and diffusion [1,2]. This behavior can be described as a random process, in which the electric characteristic considered takes on only discrete values and stochastically switches between these values. A similar behavior is usually described by the well-known random telegraph noise (RTN). The time intervals between two successive transitions are random, and the spectrum of values taken by the fluctuating quantity is discrete. In many different systems, such as semiconductor devices and $p$ - $n$ junctions, metal-oxide-semiconductor field-effect transistors (MOSFETs), point contacts and tunnel junctions, small semiconductor resistors or small metallic samples, the random switching of the resistance among different values and, consequently, of the current and voltage was observed. For a complete review about RTN, see [1]. At low temperatures, typically only one or a few telegraph processes can be observed, but increasing the temperatures (or the voltages applied to the device), the number of contributing telegraph processes increases [3-5]. At high enough temperatures, a continuous $1 / f$ noise, rather than discrete resistance switching, is observed (see [3,4,6] for two different interpretations of this behavior). According to Wakai and Van Harlingen [7], who measured the RTN in PbInAu- $\operatorname{In}_{2} \mathrm{O}_{3}-\mathrm{Pb}$ Josephson junctions, RTN is generated by electron transitions from the metallic electrodes to electron traps in the oxide layer and back. Moreover, RTN has been observed in numerous nanodevices based on semiconductors, normal metals and superconductors [3-5,8-12]. However, despite the great progress in $1 / f$ noise physics, the full understanding of the origins of low-frequency fluctuations in most of the systems showing $1 / f$ and/or random telegraph noise is still an unsolved problem.

In superconductors, fluctuations occur due to the random motion of quasi-particles, i.e., the unpaired electrons, similarly to the motion of electrons in a normal conductor. A Josephson junction (JJ) is a superconducting device composed by two superconductors separated by a thin layer of non-superconducting material that can be in a resistive state or in a superconducting state. The stationary and nonstationary Josephson relations describe the behavior of supercurrents and voltage difference across the junction as a function of the difference $\varphi$ of the phases of wavefunctions describing the condensates in the two superconductors. Moreover, JJs are widely studied in out of equilibrium systems characterized by tilted or switching periodic potentials $[13,14]$.

Recently, the characterization of JJs as detectors, based on the statistics of the escape times, has been proposed [15-22]. Specifically, the statistical analysis of the switching from the metastable superconducting state to the resistive running state of the $\mathrm{JJ}$ has been proposed to detect weak periodic signals embedded in a noisy environment [17,18]. Moreover, the rate of escape from one of the metastable wells of the tilted washboard potential of a JJ encodes information about the non-Gaussian noise present in the input signal [15,16,19-21]. After the seminal paper by Tobiska and Nazarov [23], JJs used as threshold detectors allow one to study non-Gaussian features of current noise $[19,20]$. Specifically, when a JJ leaves the metastable zero voltage state, it switches to a running resistive state, and a voltage appears across the junction. Therefore, it is possible to measure the escape times or switching times directly and to determine their probability distribution [24-29]. 
In the last decade, theoretical progress allowed calculating the entire probability distribution of the noise signal and its cumulants, performing a full counting statistics of the current fluctuations [30]. Moreover, the presence of non-Gaussian noise signals has been found experimentally in many systems [31-36]. As an example, in a wireless ad hoc network with a Poisson field of co-channel users, the noise has been well modeled by an $\alpha$-stable distribution [36]. A non-equilibrated heat reservoir can be considered as a source of non-Gaussian noise sources [33-35]. Specifically, the effect of non-Gaussian noise on the average escape time from the superconducting metastable state of a current biased JJ, coupled with non-equilibrium current fluctuations, has been experimentally investigated [31,32].

Other theoretical works analyzed the switching dynamics from the superconducting state to the resistive one. In long and short JJs, in the presence of both Gaussian and non-Gaussian noise sources, the existence of a threshold junction length has been shown above which the formation of noise-induced solitons is permitted [37-43]. The dependence of this threshold length with respect to the geometry of the junction was also studied [44], finding that for the annular structure, it is twice as long as the linear one. For a junction length smaller than this value, the connection among the parts of the junction is so strong, that soliton formation is forbidden. As a result, the junction can move from, or remain inside, a potential minimum as a whole.

In this work, we study the effects of a source of dichotomous RTN (dRTN) on a long JJ (LJJ), excited by both constant and oscillating external driving currents. We study the mean switching time (MST), that is the average time the junction takes to switch from the superconducting state to the resistive regime, as a function of various parameters of system and the noise source. The dynamics of the phase difference of the LJJ, analyzed within the sine-Gordon model $[37,38,45,46]$, is characterized by the formation and propagation of particular wave packets, called solitons $[47,48]$. Their presence is strongly related to the penetration of the magnetic flux quanta, i.e., fluxons [49,50], traveling through the junction during the switching towards the resistive state.

The paper is organized as follows. Section 2 briefly illustrates the mathematical model and the computational approach used to describe the dynamics of the system, carefully discussing the sine-Gordon (SG) equation and the dichotomous noise signal. In Section 3, results are presented and discussed. In the last section, the conclusions are drawn.

\section{The Model}

In long JJs, the magnetic field generated by the Josephson current itself is no longer negligible. Long JJs have a spatial size larger than the Josephson penetration depth $\lambda_{J}$, which gives a measure of the maximum length for which Josephson direct currents are confined at the edge of the junction. For a LJJ, the dynamics of the order parameter $\varphi$ is described in terms of a nonlinear partial differential equation, called the sine-Gordon (SG) equation. The SG equation used in our work is "perturbed" by a dissipative term, a driving current $i_{b}(t)$ and the $\mathrm{dRTN}$ term $i_{\mathrm{DN}}$, which appear in the RHS of the following equation:

$$
\beta_{c} \frac{\mathrm{d}^{2} \varphi}{\mathrm{d} t^{2}}-\frac{\mathrm{d}^{2} \varphi}{\mathrm{d} x^{2}}+\sin (\varphi)=-\frac{\mathrm{d} \varphi}{\mathrm{d} t}+i_{b}(t)+i_{\mathrm{DN}}(t)
$$

Here, the $x$-axis is along the junction and the $z$-axis is perpendicular to the barrier. Equation (1) is written measuring the distance $x$ in units of $\lambda_{J}$ and the time $t$ in units of the inverse of the characteristic frequency 
$\omega_{J}$ of the junction. The coefficient $\beta_{c}=\omega_{J} R C$, where $R$ and $C$ are the effective normal resistance and capacitance of the junction, respectively, is the Stewart-McCumber parameter. The bias current $i_{b}(t)$ and the supercurrent are both normalized to the critical current $i_{c}$ of the junction. The presence of an external magnetic field $\Gamma$ is taken into account in the boundary conditions of Equation (1):

$$
\varphi_{x}(0, t)=\varphi_{x}(L, t)=\Gamma
$$

where $L$ is the length of the junction normalized to $\lambda_{J}$. Hereinafter $\Gamma=0$ is imposed. An LJJ can be depicted as a long string lying along the washboard potential (WP) $U(\varphi, x, t)$ given by:

$$
U(\varphi, x, t)=1-\cos (\varphi(x, t))-i_{b}(t) \varphi(x, t)
$$

The sinusoidal term of Equation (3) gives, at each time, the sequence of the maxima and minima (metastable states) characteristics of the WP, while the bias current $i_{b}(t)$ determines the slope of the potential. A tilted washboard potential and a string located between two consecutive valleys are shown in (a) of Figure 1.
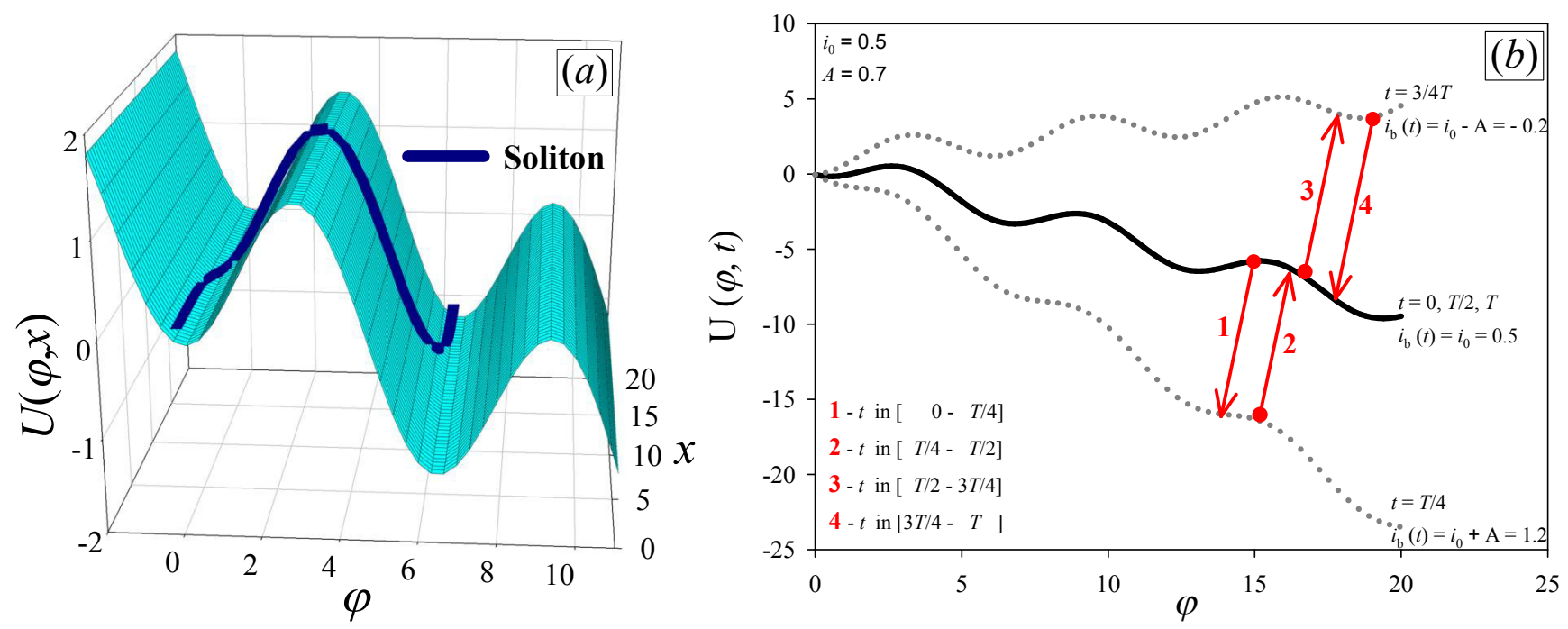

Figure 1. (a) Washboard potential in a fixed instant of its dynamics with a soliton located between two adjacent valleys. (b) Different slopes of the washboard potential during one period $T$ of oscillation, in particular at times $t=0, T / 2, T$ (solid line) and $t=T / 4,3 T / 4$ (dotted lines), with $i_{0}=0.5$ and $A=0.7$. The red arrows are used to stress the four different "parts" in which the time evolution of the potential can be divided. This partition allows us to identify the time intervals in which the "rightward" escape dynamics of the string is supported or disadvantaged by the dynamics of the potential.

The external bias current is formed by a constant term $i_{0}$ in addition to an oscillating component:

$$
i_{b}(t)=i_{0}+A \sin (\omega t)
$$

where the amplitude $A$ and the frequency $\omega$ of the dimensionless driving current are normalized to $i_{c}$ and $\omega_{J}$, respectively. Hereafter, the amplitude of the oscillating component of the current is set to $A=0.7$. Due to the sinusoidal term in the bias current, the washboard slope oscillates with the same frequency 
$\omega$ around the mean value $i_{0}$. When $i_{b} \geq 1$, the barriers between consecutive minima of the potential vanish, and the metastable states are removed.

The expression for the left (or right) potential barrier height $\Delta U^{+}$(or $\Delta U^{-}$) is:

$$
\Delta U^{ \pm}(t)=2 \sqrt{1-i_{b}^{2}(t)}+i_{b}(t)\left[2 \arcsin \left(i_{b}(t)\right) \pm \pi\right]
$$

In this work, three different initial values of the bias current are set, namely $i_{0}=\{0.1,0.5,0.9\}$, so that the time averages of the right potential barrier height are $\overline{\Delta U_{i_{0}}^{-}} \simeq\{1.9,1.0,0.4\}$, respectively.

The SG equation admits stable traveling wave solutions, called solitons and antisolitons, represented by a $2 \pi$ step in $\varphi$ with the string lying on two adjacent WP valleys (see the solid line in (a) of Figure 1). The rest energy of a soliton is equal to eight in units of $E_{0}$, where $E_{0}$ is defined as the Josephson coupling energy of a small Josephson junction of area $\lambda_{J} w$, with $w$ the width of the junction. In a long $\mathrm{JJ}$, all energies are normalized to the characteristic energy $E_{0}$, which is obtained as the product between the Josephson coupling energy per unit length $e_{J}[50]$ and the Josephson penetration length $\lambda_{J}$, that is $E_{0}=e_{J} \lambda_{J}$. In Equation (5), $\Delta U^{ \pm}$are the energy barriers seen by every cell (i.e., the short junction limit of a long JJ) normalized to the Josephson coupling energy $e_{J}$ [29].

The soliton formation and the MST behavior are studied looking at the escape dynamics of the string through the potential barriers, whose heights are just $\Delta U^{ \pm}$. The string is initially at rest within the first washboard valley, that is $\varphi(x, 0)=\arcsin \left(i_{0}\right) \quad \forall x: 0 \leq x \leq L$.

The stochastic current $i_{\mathrm{DN}}(x, t)$, representing the dRTN signal, is equal to:

$$
i_{\mathrm{DN}}(x, t)=\gamma \beta(t)
$$

where $\gamma$ is the normalized noise amplitude and $\beta(t)$ randomly jumps between two values, $\beta_{\text {up }}$ and $\beta_{\text {down }}$. In detail, the parameter $\beta(t)$ is a dichotomous stochastic process, whose jump rate is a periodic function:

$$
\gamma_{\mathrm{DN}}(t)= \begin{cases}0, & \Delta t_{r} \leq \tau_{\mathrm{d}} \\ \gamma_{0}\left(1+A_{\mathrm{DN}}\left|\cos \left(\omega_{\mathrm{DN}} t\right)\right|\right), & \Delta t_{r}>\tau_{\mathrm{d}} .\end{cases}
$$

Here, $\Delta t_{r}$ is the random time interval between two consecutive switches, and $\tau_{\mathrm{d}}$ is the delay between two jumps, that is the time interval after a switch, before another jump can occur. In Equation (7), $A_{\mathrm{DN}}$ and $\omega_{\text {DN }}$ are the amplitude and the angular frequency of the periodic term, respectively, and $\gamma_{0}$ is the jump rate in the absence of periodic term. In Figure 2, the time series of $\beta(t)$ for different values of the delay $\tau_{\mathrm{d}}$, namely $\tau_{\mathrm{d}}=0.5,3,10$, is shown, setting $\beta_{\text {up }}=1$ and $\beta_{\text {down }}=-1$. We note that the correlation time $\tau_{\mathrm{d}}$ of the dichotomous noise affects the time of switching between the two levels of $\beta(t)$. For a delay time greater than $T_{\mathrm{DN}}$, where $T_{\mathrm{DN}}=2 \pi / \omega_{\mathrm{DN}}$, we observe a synchronization between the jumps and the periodicity of the rate $\gamma_{\mathrm{DN}}(t)$. A similar approach to model the dichotomous noise source was previously implemented and used in other contexts, from a Lotka-Volterra system of two [51,52] or more [53,54] competing species to electron transport dynamics in Gallium arsenide (GaAs) samples [55]. 

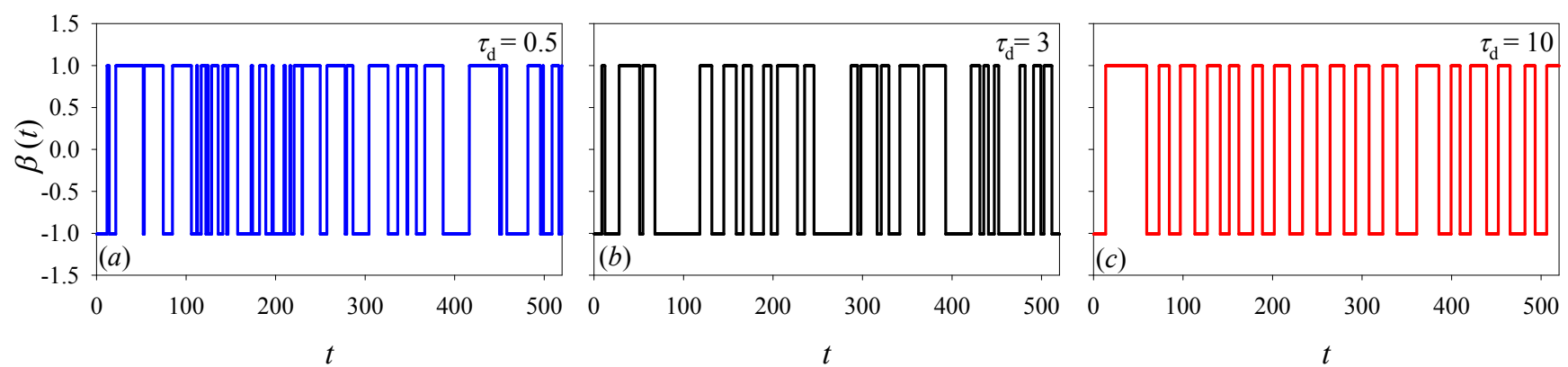

Figure 2. Time evolution of the dichotomous noise signal $\beta(t)$ with different values of the delay: $\tau_{\mathrm{d}}=0.5(\mathbf{a}), 3(\mathbf{b}), 10(\mathbf{c})$. The values of the other parameters are: $A_{\mathrm{DN}}=0.9$, $\omega_{\mathrm{DN}}=1.0, \gamma_{0}=0.02, \beta(0)=\beta_{\text {down }}$.

Equation (1) is numerically solved within the Ito scheme. The system is overdamped, condition obtained imposing $\beta_{c}=0.01$. The numerical solution of Equation (1) is calculated setting the time and spatial steps to $\Delta t=0.05$ and $\Delta x=0.05$, respectively. We calculate the mean switching time (MST) towards the resistive state, starting from the metastable state (bottom of a potential minimum) corresponding to the superconducting regime. The MST $\tau$ is a nonlinear relaxation time (NLRT) [56] and represents the mean value of the permanence times of the phase $\varphi$ within the first valley, that is $\varphi \in\left[\varphi_{\text {Max }}^{L}, \varphi_{\text {Max }}^{R}\right]$. Due to the stochastic nature of the dynamics of the system, the switching time has been obtained averaging over a sufficiently large number $(N=5000)$ of stochastic realizations. The thresholds $\varphi_{M a x}^{L}$ and $\varphi_{M a x}^{R}$ are, respectively, the positions of the left and right maxima, which bound the minimum chosen as the initial condition. No absorbing barriers are set, so that during the entire observation time, $t_{\max }$, all of the events of permanence inside the initial minimum are taken into account to calculate $\tau$. The probability $P_{i j}$ that $\varphi \in\left[\varphi_{\text {Max }}^{L}, \varphi_{\text {Max }}^{R}\right]$, in the $i$-th realization for the $j$-th cell (i.e., the elementary parts forming the string), is:

$$
P_{i j}(t)=\left\{\begin{array}{l}
1 \Longleftrightarrow \varphi \in\left[\varphi_{\text {Max }}^{L}, \varphi_{\text {Max }}^{R}\right] \\
0 \Longleftrightarrow \varphi \notin\left[\varphi_{\text {Max }}^{L}, \varphi_{\text {Max }}^{R}\right] .
\end{array}\right.
$$

Summing $P_{i j}(t)$ over the total number $N_{c}=L / \Delta x$ of cells and over the number $N$ of realizations, the average probability that the entire string is in the superconducting state at time $t$ can be computed:

$$
P(t)=\frac{1}{N N_{c}} \sum_{i=1}^{N} \sum_{j=1}^{N_{c}} P_{i j}(t) .
$$

The MST $\tau$ is therefore calculated as:

$$
\tau=\int_{0}^{t_{\max }} P(t) d t
$$

\section{Results}

The analysis is performed studying the MST $\tau$ as a function of the noise intensity $\gamma$. The values of $\gamma$ are in the interval $\gamma \in\left[10^{-4}, 10^{2}\right]$ and normalized to the critical current $i_{c}$. The length of the junction is set to $L=20$, that is a value large enough to allow solitons formation along the string [37,43]. The 
driving frequency is set to $\omega=0.2$. Three different values of the correlation time $\tau_{\mathrm{d}}$ are used, namely $\tau_{\mathrm{d}}=\{0.5,3,10\}$ (see Figure 2). These values are chosen to be, respectively, shorter, comparable and greater than half oscillation period $T_{\mathrm{DN}} / 2$ of the jump rate $\gamma_{\mathrm{DN}}(t)$ of the noise signal (see Equation (7)).

The escape dynamics from the metastable state is strongly related to the height of the potential barriers that the phase string has to overcome to switch towards the resistive state. The height of these barriers, according to the Equation (5), is directly dependent on the slope of the potential, which coincides with the bias current value. To completely explore the switching dynamics of an LJJ, three values of the initial bias current are chosen $i_{0}=\{0.1,0.5,0.9\}$. In this way, we set, respectively, a small, halfway and high value of the mean potential slope. Using a large positive initial bias current, the escape events from the metastable state occur preferentially throughout the "right" potential barrier. Conversely, setting small values of the initial bias current, the escape dynamics throughout the "left" potential barrier can also occur.
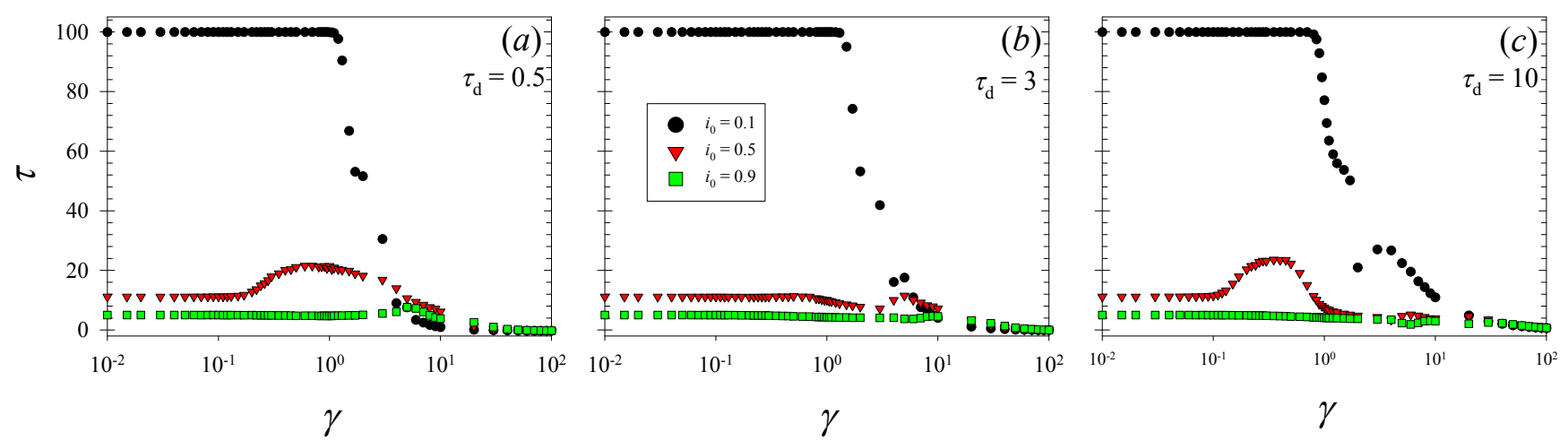

Figure 3. Mean switching time (MST) $\tau$ as a function of the noise intensity $\gamma$, varying the initial bias current $i_{0}=\{0.1,0.5,0.9\}$ and the correlation time $\tau_{\mathrm{d}}=\{0.5,3,10\}$ with a random initial value of $\beta(t)$. The values of $\tau_{\mathrm{d}}$ are: $0.5(\mathbf{a}), 3(\mathbf{b})$ and $10(\mathbf{c})$. The legend in (b) refers to all panels.

The dRTN signal of Equation (6), applied to the junction together with an external bias current, can hinder or support the switching dynamics from the superconducting state. In particular, a noise contribution with the sign equal to the bias current increases the potential slope, assisting the escape events of the phase string. Conversely, a noise contribution of opposite sign to the bias current contrasts with the escape events and further confines the string within a potential minimum. Therefore, an initial value of the dichotomous noise of the sign equal or opposite to the bias current can strongly affect the MST value. Although the MST $\tau$ is an NLRT, the value of $\tau$ is mainly influenced by the time the string requires for the first escape from the initial metastable state. Therefore, imposing $\beta(0)=\beta_{\text {down }}$ or $\beta(0)=\beta_{u p}$, the phase evolution of the string and the value of $\tau$ can drastically change. To release the results from the initial condition of the dRTN, the value of $\beta(0)$ can be randomly chosen among $\beta_{\text {down }}$ and $\beta_{u p}$ in each numerical realization. The data obtained randomly choosing the initial value $\beta(0)$ of the dichotomous signal are shown in Figure 3. Panels (a), (b) and (c) of this picture show data for $\tau_{\mathrm{d}}=\{0.5,3,10\}$, respectively, and every panel contains curves for $i_{0}=\{0.1,0.5,0.9\}$. The $\tau$ data for $i_{0}=0.1$ show an evident saturation effect for low noise intensities as the result of setting an observation time $t_{\max }$ not long enough to observe escape events. Nonmonotonic behaviors characterize the MST 
curves. To well understand the results obtained, the analysis setting $\beta(0)=\beta_{\text {down }}$ and $\beta(0)=\beta_{\text {up }}$ had to be performed.

The results of this study are shown in Figure 4. Every panel of this figure contains curves for $i_{0}=\{0.1,0.5,0.9\}$. The top panels are obtained setting $\beta(0)=\beta_{\text {down }}$ with $\tau_{\mathrm{d}}=\{0.5$ (a), 3 (b) and 10 (c) $\}$. The bottom panels are obtained setting $\beta(0)=\beta_{u p}$ with $\tau_{\mathrm{d}}=\{0.5$ (d), 3 (e) and 10 (f) $\}$.
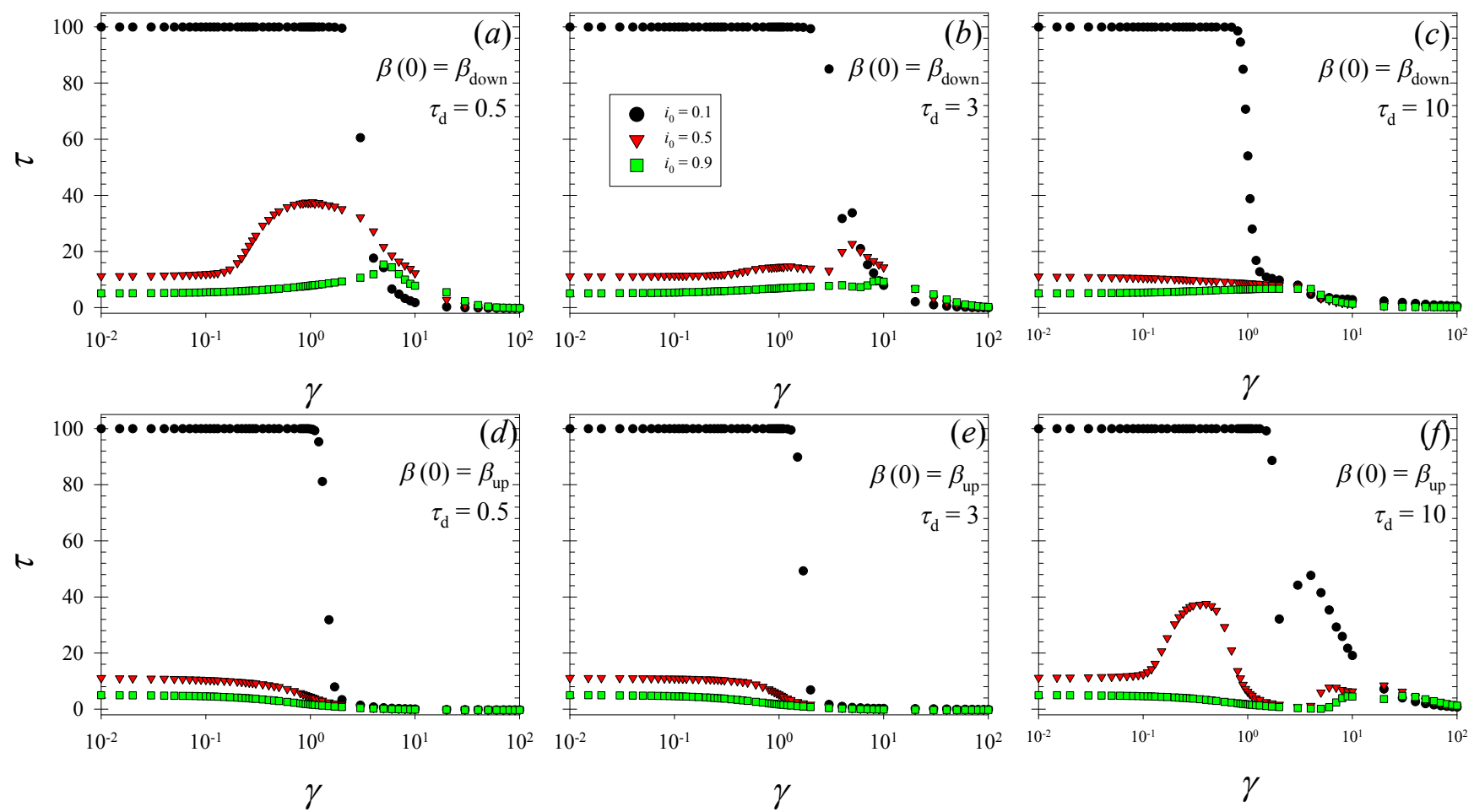

Figure 4. MST $\tau$ as a function of the noise intensity $\gamma$, with initial bias current $i_{0}=$ $\{0.1,0.5,0.9\}$ and correlation time $\tau_{\mathrm{d}}=\{0.5,3,10\}$, setting the initial values of $\beta(t)$ to $\beta(0)=\beta_{\text {down }}(\mathbf{a}, \mathbf{b}, \mathbf{c})$ and $\beta(0)=\beta_{\text {up }}(\mathbf{d}, \mathbf{e}, \mathbf{f})$. The values of $\tau_{\mathrm{d}}$ are: $0.5(\mathrm{a}, \mathrm{d}) ; 3(\mathrm{~b}, \mathrm{e}) ; 10(\mathbf{c}, \mathbf{f})$. The driving frequency is $\omega=0.2$. The legend in (b) refers to all panels.

All curves for $i_{0}=0.1$ show a saturation effect of the MST (i.e., $\tau=t_{\max }$ ) for low noise intensities, followed by a decreasing behavior. Setting this slope $\left(i_{0}=0.1\right)$, the mean values of the right and left potential barrier are $\overline{\Delta U_{0.1}^{-}} \simeq 1.9$ and $\overline{\Delta U_{0.1}^{+}} \simeq 2.6$, respectively. The cells can escape from the potential minimum only when the noise contribution is strong enough to cancel the potential barriers, that is when $\gamma>1$.

With $i_{0}=0.5$ and $i_{0}=0.9$, the bias current $i_{b}(t)$ takes on values greater than one, so that the right potential barrier vanishes during the oscillation of the potential. As a result, the string is able to escape from the potential minimum also if $\gamma \rightarrow 0$. In particular, for $i_{0}=0.5$ and $i_{0}=0.9$, the MST are $\tau_{\gamma \rightarrow 0}^{0.5} \simeq 11.4$ and $\tau_{\gamma \rightarrow 0}^{0.9} \simeq 5.3$, respectively, for each value of $\tau_{\mathrm{d}}$ and both initial conditions.

It is noteworthy that in Panels (a), (b) and (c) of Figure 4 for high noise intensities, specifically for $\gamma \gtrsim 4$, the curve for $i_{0}=0.1$ is lower than the curves for greater slopes. This counterintuitive behavior is related to the initial condition $\beta(0)=\beta_{\text {down }}$ and to the fact that $\tau$ is computed as an NLRT. A very intense noise in fact causes a rapid shift leftward of the string. However, when a mean slope significantly greater than zero is applied, i.e., $i_{0}=0.5$ and 0.9 , the string tends to roll rightward along the potential 
up to returning within the initial potential valley, contributing again to increasing the MST. The data of the bottom Panels (d), (e) and (f) of Figure 4, obtained setting $\beta(0)=\beta_{u p}$, show that the MST, as well as the right potential barrier, reduces as $i_{0}$ increases.

The data obtained for $i_{0}=0.5$, shown in (a) of Figure 4, as the noise intensity increases, exhibit an effect of noise-enhanced stability (NES) [42,43,56-74], a noise-induced phenomenon consisting of a nonmonotonic behavior with the appearance of a maximum. In the presence of NES, the stability of metastable states is enhanced, and the average lifetime of the metastable state increases non-monotonically with the noise intensity. The maximum shown in Panel (a) is obtained in correspondence with $\gamma_{\mathrm{NES}}=1$ and $\tau_{\mathrm{NES}} \simeq 38$. This NES effect occurs due to several negative and positive jumps that the dRTN source exhibits for a low value of $\tau_{\mathrm{d}}$, before the string escapes from the metastable state. The nodal point to observe this NES maximum is to impose as the initial condition $\beta(0)=\beta_{\text {down }}$. In this way, in the short time $\tau_{\gamma \rightarrow 0}^{0.5} \simeq 11.4$ that the string needs to escape in the absence of noise (deterministic escape time), the number of negative jumps is larger than the positive ones. As a consequence, the string is confined in the initial minimum. Conversely, setting $\beta(0)=\beta_{u p}$, for short delay times $\tau_{\mathrm{d}}$, see (d) and (e) of Figure 4, the NES maxima vanish.

Concerning the results obtained for $\beta(0)=\beta_{\text {down }}$ and $i_{0}=0.5$, shown in (b) and (c) of Figure 4, we note that, as $\tau_{\mathrm{d}}$ increases, the noise is less and less able to confine the string within the first WP minimum, and the NES effect tends to vanish. For $\tau_{\mathrm{d}}=3$, the NES maximum is centered at $\gamma=5$, and the MST value is $\tau_{\mathrm{NES}} \simeq 23$. There is no evidence of the NES effect setting $\tau_{\mathrm{d}}=10$. This occurs because up to $t=\tau_{\mathrm{d}}$, the string feels the confining effect of $\beta(0)=\beta_{\text {down }}$ during the initial times of its dynamics, that is when its inertial mass is quite high, since the string is initially at rest and the system is overdamped. For $t \gtrsim \tau_{\mathrm{d}}=10$, the RTN switches to $\beta_{u p}$, and the string feels a noise signal that assists its escape dynamics. When this occurs, the potential is in an ideal configuration to support an escape event. Panel (b) of Figure 1 shows the WP for $i_{0}=0.5$ in three different positions during one period of oscillation. The solid line of this panel represents the WP for $t=\{0, T / 2, T\}$ (mean slope), while the dotted lines represent the WP for $t=T / 4$ and $t=3 T / 4$ (maximum and minimum slopes, respectively). Setting $\omega=0.2$, a quarter of period of oscillation is $T / 4 \sim 8$. The red arrows are used to divide into four parts the evolution of the potential. The rightward escape of the phase string can be supported (Parts 1 and 2 ) or disadvantaged (Parts 3 and 4 ) by the potential dynamics. When $t \gtrsim 10, \beta(t)$ switches to $\beta_{\text {up }}$, the potential is in the second part of its evolution, and the string can be easily pushed out of the metastable state. Conversely, setting $\beta(0)=\beta_{u p}$, initially, the dRTN tends to push out the string when its inertial mass is high. When $t \gtrsim 10, \beta(t)$ switches to $\beta_{\text {down }}$, and the noise signal tends to confine the string within the potential minimum. This explains the NES effect that emerges in (f) of Figure 4 for $i_{0}=0.5$. This NES maximum is centered at $\gamma \simeq 0.4$ with an MST value $\tau_{\mathrm{NES}} \simeq 38$. The curve for $i_{0}=0.1$ of this panel shows a minimum centered at $\gamma=2$, that is in correspondence with a noise intensity that almost matches the time average of the right potential barrier height $\overline{\Delta U_{0.1}^{-}} \simeq 1.9$. For noise intensities that are a little higher, first, the MST slightly grows due to retrapping events throughout the left potential barrier and then decreases exponentially when the noise intensity further increases.

The curves shown in Figure 3 were obtained by randomly choosing the initial condition $\beta(0)$ of the dichotomous noise and are therefore an average between the results obtained setting $\beta(0)=\beta_{\text {down }}$ and those obtained for $\beta(0)=\beta_{u p}$. 


\section{Conclusions}

We studied the effects of a correlated dichotomous noise on the switching dynamics from the superconducting state towards the resistive state of a long overdamped Josephson junction (JJ). The analysis was computationally performed within the framework of the sine-Gordon equation, studying the mean lifetime of the metastable state of the junction. The mean switching time (MST) of the phase difference across the $\mathrm{JJ}$ was analyzed varying the intensity of the noise source when the system is driven by an external oscillating bias current. The junction length is set to a value great enough to observe noise-induced soliton formation along the string.

We found nonmonotonic behavior of the MST $\tau$ as a function of the noise intensity $\gamma$, with characteristics related to the initial value of the applied bias current. In particular, we observed a noise-induced phenomenon called noise-enhanced stability (NES), whose characteristics depend on the initial value imposed by the dichotomous noise. In particular, we observed several temporary trapping events related to the specific choice, $\beta_{u p}$ or $\beta_{\text {down }}$, of the initial value $\beta(0)$ of the noise source. The study was extended, exploring the effects of the correlation time $\tau_{\mathrm{d}}$ of the dichotomous noise on the nonmonotonic behaviors observed. Moreover, curves obtained for a random initial condition of the noise signal were presented. These results show non-monotonic trends that can be understood in light of the analysis performed by deterministically choosing the initial condition of $\beta(t)$.

Our results contribute to a deeper comprehension of the physics of fluctuations in long JJs in view of an improvement of the performance of these devices. Our findings could help to shed light on the general context of the nonequilibrium statistical mechanics. In fact, JJs are good candidates to probe relevant physics issues in metastable systems [25]. Moreover, the MST from one of the metastable states of the potential profile encodes information on the non-Gaussian background noise. Therefore, the statistical analysis of the switching times of JJs can be used to analyze weak signals in the presence of a background noise characterized by unknown non-Gaussian statistics.

\section{Acknowledgments}

This work was supported by MIUR (Ministero dell'Istruzione, dell'Università e della Ricerca) through Grant No. PON02_00355_3391233, “Tecnologie per l'ENERGia e l'Efficienza energETICa-ENERGETIC".

\section{Author Contributions}

All authors contributed to the conception of the study, obtained and interpreted the results and the preparation of this work. All authors have read and approved the final manuscript.

\section{Conflicts of Interest}

The authors declare no conflict of interest. 


\section{References}

1. Kogan, S. Electronic Noise and Fluctuations in Solids; Cambridge University Press: Cambridge, UK, 1996.

2. Pankratov, E.; Spagnolo, B. Optimization of impurity profile for p-n-junction in heterostructures. Eur. Phys. J. B 2005, 46, 15-19.

3. Rogers, C.; Buhrman, R. Composition of $1 / f$ Noise in Metal-Insulator-Metal Tunnel Junctions. Phys. Rev. Lett. 1984, 53, 1272-1275.

4. Rogers, C.; Buhrman, R. Nature of single-localized-electron states derived from tunneling measurements. Phys. Rev. Lett. 1985, 55, 859-862.

5. Ralls, K.; Skocpol, W.; Jackel, L.; Howard, R.; Fetter, L.; Epworth, R.; Tennant, D. Discrete Resistance Switching in Submicrometer Silicon Inversion Layers: Individual Interface Traps and Low-Frequency 1/f Noise. Phys. Rev. Lett. 1984, 52, 228-231.

6. Ralls, K.S.; Buhrman, R.A. Microscopic study of $1 / f$ noise in metal nanobridges. Phys. Rev. $B$ 1991, 44, 5800-5817.

7. Wakai, R.; van Harlingen, D. Direct lifetime measurements and interactions of charged defect states in submicron Josephson junctions. Phys. Rev. Lett. 1987, 58, 1687-1690.

8. Parman, C.; Israeloff, N.; Kakalios, J. Random telegraph-switching noise in coplanar current measurements of amorphous silicon. Phys. Rev. B 1991, 44, 8391-8394.

9. Peters, M.; Dijkhuis, J.; Molenkamp, L. Random telegraph signals and $1 / f$ noise in a silicon quantum dot. J. Appl. Phys. 1999, 86, 1523-1526.

10. Gunnarsson, D.; Duty, T.; Bladh, K.; Delsing, P. Tunability of a $2 e$ periodic single Cooper pair box. Phys. Rev. B 2004, 70, 224523.

11. Eroms, J.; van Schaarenburg, L.; Driessen, E.; Plantenberg, J.; Huizinga, C.; Schouten, R.; Verbruggen, A.; Harmans, C.; Mooij, J. Low-frequency noise in Josephson junctions for superconducting qubits. Appl. Phys. Lett. 2006, 89, 122516.

12. Paladino, E.; Galperin, Y.; Falci, G.; Altshuler, B. $1 / f$ noise: Implications for solid-state quantum information. Rev. Mod. Phys. 2014, 86, 361-418.

13. Reimann, P.; van den Broeck, C.; Linke, H.; Hänggi, P.; Rubi, J.; Pérez-Madrid, A. Giant acceleration of free diffusion by use of tilted periodic potentials. Phys. Rev. Lett. 2001, 87, 010602.

14. Dubkov, A.A.; Spagnolo, B. Acceleration of diffusion in randomly switching potential with supersymmetry. Phys. Rev. E 2005, 72, 041104.

15. Grabert, H. Theory of a Josephson junction detector of non-Gaussian noise. Phys. Rev. B 2008, 77, 205315.

16. Urban, D.; Grabert, H. Feedback and rate asymmetry of the Josephson junction noise detector. Phys. Rev. B 2009, 79, 113102.

17. Filatrella, G.; Pierro, V. Detection of noise-corrupted sinusoidal signals with Josephson junctions. Phys. Rev. E 2010, 82, 046712.

18. Addesso, P.; Filatrella, G.; Pierro, V. Characterization of escape times of Josephson junctions for signal detection. Phys. Rev. E 2012, 85, 016708. 
19. Ankerhold, J. Detecting charge noise with a Josephson junction: A problem of thermal escape in presence of non-Gaussian fluctuations. Phys. Rev. Lett. 2007, 98, 036601.

20. Sukhorukov, E.V.; Jordan, A.N. Stochastic dynamics of a Josephson junction threshold detector. Phys. Rev. Lett. 2007, 98, 136803.

21. Köpke, M.; Ankerhold, J. Linear dynamics subject to thermal fluctuations and non-Gaussian noise: From classical to quantum. New J. Phys. 2013, 15, 043013.

22. Oelsner, G; Revin, L.S.; Il'Ichev, E.; Pankratov, A.L.; Meyer, H.G.; Grönberg, L.; Hassel, J.; Kuzmin, L.S. Underdamped Josephson junction as a switching current detector. Appl. Phys. Lett. 2013, 14, 142605.

23. Tobiska, J.; Nazarov, Y.V. Josephson junctions as threshold detectors for full counting statistics. Phys. Rev. Lett. 2004, 93, 106801.

24. Yu, Y.; Han, S. Resonant escape over an oscillating barrier in underdamped Josephson tunnel junctions. Phys. Rev. Lett. 2003, 91, 127003.

25. Sun, G.; Dong, N.; Mao, G.; Chen, J.; Xu, W.; Ji, Z.; Kang, L.; Wu, P.; Yu, Y.; Xing, D. Thermal escape from a metastable state in periodically driven Josephson junctions. Phys. Rev. E 2007, 75, 021107.

26. Pan, C.; Tan, X.; Yu, Y.; Sun, G.; Kang, L.; Xu, W.; Chen, J.; Wu, P. Resonant activation through effective temperature oscillation in a Josephson tunnel junction. Phys. Rev. E 2009, 79, 030104.

27. Devoret, M.H.; Martinis, J.M.; Esteve, D.; Clarke, J. Resonant activation from the zero-voltage state of a current-biased Josephson junction. Phys. Rev. Lett. 1984, 53, 1260-1263.

28. Devoret, M.H.; Martinis, J.M.; Clarke, J. Measurements of macroscopic quantum tunneling out of the zero-voltage state of a current-biased Josephson junction. Phys. Rev. Lett. 1985, 55, 1908-1911.

29. Castellano, M.; Torrioli, G.; Cosmelli, C.; Costantini, A.; Chiarello, F.; Carelli, P.; Rotoli, G.; Cirillo, M.; Kautz, R. Thermally activated escape from the zero-voltage state in long Josephson junctions. Phys. Rev. B 1996, 54, 15417-15428.

30. Novotnỳ, T. Josephson junctions as threshold detectors of full counting statistics: Open issues. J. Stat. Mech. Theory Exp. 2009, P01050.

31. Huard, B.; Pothier, H.; Birge, N.O.; Esteve, D.; Waintal, X.; Ankerhold, J. Josephson junctions as detectors for non-Gaussian noise. Ann. Phys. 2007, 16, 736-750.

32. Peltonen, J.; Timofeev, A.; Meschke, M.; Heikkilä, T.; Pekola, J. Detecting non-Gaussian current fluctuations using a Josephson threshold detector. Physica E 2007, 40, 111-122.

33. Montroll, E.W.; Shlesinger, M.F. Nonequilibrium Phenomena II: From Stochastics to Hydrodynamics; North-Holland, Amsterdam, 1984; pp. 1-121.

34. Shlesinger, M.F.; Zaslavsky, G.M.; Frisch, U. Lévy Flights and Related Topics in Physics; Springer: Berlin/Heidelberg, Germany, 1994; Volume 450.

35. Dybiec, B.; Gudowska-Nowak, E. Resonant activation in the presence of nonequilibrated baths. Phys. Rev. E 2004, 69, 016105.

36. Souryal, M.R.; Larsson, E.G.; Peric, B.; Vojcic, B.R. Soft-decision metrics for coded orthogonal signaling in symmetric alpha-stable noise. IEEE Trans. Signal Process. 2008, 56, 266-273. 
37. Fedorov, K.; Pankratov, A. Mean time of the thermal escape in a current-biased long-overlap Josephson junction. Phys. Rev. B 2007, 76, 024504.

38. Fedorov, K.; Pankratov, A.L.; Spagnolo, B. Influence of length on the noise delayed switching of long Josephson junctions. Int. J. Bifurc. Chaos 2008, 18, 2857-2862.

39. Augello, G.; Valenti, D.; Spagnolo, B. Effects of colored noise in short overdamped Josephson junction. Int. J. Bifurc. Chaos 2008, 6, 801-806.

40. Augello, G.; Valenti, D.; Pankratov, A.L.; Spagnolo, B. Lifetime of the superconductive state in short and long Josephson junctions. Eur. Phys. J. B 2009, 70, 145-151.

41. Augello, G.; Valenti, D.; Spagnolo, B. Non-Gaussian noise effects in the dynamics of a short overdamped Josephson junction. Eur. Phys. J. B 2010, 78, 225-234.

42. Guarcello, C.; Valenti, D.; Augello, G.; Spagnolo, B. The Role of Non-Gaussian Sources in the Transient Dynamics of Long Josephson Junctions. Acta Phys. Pol. B 2013, 44, 997-1005.

43. Valenti, D.; Guarcello, C.; Spagnolo, B. Switching times in long-overlap Josephson junctions subject to thermal fluctuations and non-Gaussian noise sources. Phys. Rev. B 2014, 89, 214510.

44. Fedorov, K.G.; Pankratov A.L. Crossover of the thermal escape problem in annular spatially distributed systems. Phys. Rev. Lett. 2009, 103, 260601.

45. Barone, A.; Paterno, G. Physics and Applications of the Josephson Effect; Wiley: Hoboken, NJ, USA, 1982.

46. Likharev, K. Dynamics of Josephson Junctions and Circuits; Gordon \& Breach: New York, NY, USA, 1986.

47. Ustinov, A. Solitons in Josephson junctions. Physica D 1998, 123, 315-329.

48. Büttiker, M.; Landauer, R. Nucleation theory of overdamped soliton motion. Phys. Rev. A 1981, 23, 1397-1410.

49. McLaughlin, D.W.; Scott, A.C. Perturbation analysis of fluxon dynamics. Phys. Rev. A 1978, $18,1652-1680$.

50. Dueholm, B.; Joergensen, E.; Levring, O.; Monaco, R.; Mygind, J.; Pedersen, N.; Samuelsen, M. An analysis of fluxons in long Josephson junctions. IEEE Trans. Magn. 1983, 19, 1196-1200.

51. Valenti, D.; Schimansky-Geier, L.; Sailer, X.; Spagnolo, B. Moment equations for a spatially extended system of two competing species. Eur. Phys. J. B 2006, 50, 199-203.

52. Valenti, D.; Schimansky-Geier, L.; Sailer, X.; Spagnolo, B.; Iacomi, M. Moment Equations in a Lotka-Volterra Extended System with Time Correlated Noise. Acta Phys. Pol. B 2007, 38, 1961-1972.

53. Valenti, D.; Spagnolo, B. Stochastic dynamics and mean field approach in a system of three interacting species. Cent. Eur. J. Phys. 2009, 7, 457-471.

54. Valenti, D.; Pizzolato, N.; Spagnolo, B. Mean Field Approach and Role of the Coloured Noise in the Dynamics of Three Interacting Species. Acta Phys. Pol. B 2010, 41, 1051-1071.

55. Adorno, D.P.; Pizzolato, N.; Valenti, D.; Spagnolo, B. External Noise Effects in Doped Semiconductors Operating Under sub-THz Signals. Rep. Math. Phys. 2012, 70, 171-179.

56. Dubkov, A.A.; Agudov, N.V.; Spagnolo, B. Noise-enhanced stability in fluctuating metastable states. Phys. Rev. E 2004, 69, 061103. 
57. Mantegna, R.; Spagnolo, B. Noise Enhanced Stability in an Unstable System. Phys. Rev. Lett. 1996, 76, 563-566.

58. Agudov, N.; Spagnolo, B. Noise-enhanced stability of periodically driven metastable states. Phys. Rev. E 2001, 64, 035102.

59. Agudov, N.V.; Dubkov, A.A.; Spagnolo, B. Escape from a metastable state with fluctuating barrier. Physica A 2003, 325, 144-151.

60. Spagnolo, B.; Agudov, N.; Dubkov, A. Noise enhanced stability. Acta Phys. Pol. B 2004, 35, 1419-1436.

61. Spagnolo, B.; Dubkov, A.; Agudov, N. Enhancement of stability in randomly switching potential with metastable state. Eur. Phys. J. B 2004, 40, 273-281.

62. D’Odorico, P.; Laio, F.; Ridolfi, L. Noise-induced stability in dryland plant ecosystems. Proc. Natl. Acad. Sci. USA 2005, 102, 10819-10822.

63. Fiasconaro, A.; Spagnolo, B.; Boccaletti, S. Signatures of noise-enhanced stability in metastable states. Phys. Rev. E 2005, 72, 061110

64. Hurtado, P.I.; Marro, J.; Garrido, P. Metastability, nucleation, and noise-enhanced stabilization out of equilibrium. Phys. Rev. E 2006, 74, 050101.

65. Spagnolo, B.; Dubkov, A.; Pankratov, A.; Pankratova, E.; Fiasconaro, A.; Ochab-Marcinek, A. Lifetime of metastable states and suppression of noise in interdisciplinary physical models. Acta Phys. Pol. B 2007, 38, 1925-1950.

66. Bonanno, G.; Valenti, D.; Spagnolo, B. Mean escape time in a system with stochastic volatility. Phys. Rev. E 2007, 75, 016106.

67. Mankin, R.; Soika, E.; Sauga, A.; Ainsaar, A. Thermally enhanced stability in fluctuating bistable potentials. Phys. Rev. E 2008, 77, 051113.

68. Yoshimoto, M.; Shirahama, H.; Kurosawa, S. Noise-induced order in the chaos of the Belousov-Zhabotinsky reaction. J. Chem. Phys. 2008, 129, 014508.

69. Valenti, D.; Augello, G.; Spagnolo, B. Dynamics of a FitzHugh-Nagumo system subjected to autocorrelated noise. Eur. Phys. J. B 2008, 65, 443-451.

70. Fiasconaro, A.; Spagnolo, B. Stability measures in metastable states with Gaussian colored noise. Phys. Rev. E 2009, 80, 041110.

71. Trapanese, M. Noise enhanced stability in magnetic systems. J. Appl. Phys. 2009, 105, 07 D313.

72. Fiasconaro, A.; Mazo, J.J.; Spagnolo, B. Noise-induced enhancement of stability in a metastable system with damping. Phys. Rev. E 2010, 82, 041120.

73. Li, J.h.; Łuczka, J. Thermal-inertial ratchet effects: Negative mobility, resonant activation, noise-enhanced stability, and noise-weakened stability. Phys. Rev. E 2010, 82, 041104.

74. Smirnov, A.A.; Pankratov, A.L. Influence of the size of uniaxial magnetic nanoparticle on the reliability of high-speed switching. Phys. Rev. B 2010, 82, 132405.

(c) 2015 by the authors; licensee MDPI, Basel, Switzerland. This article is an open access article distributed under the terms and conditions of the Creative Commons Attribution license (http://creativecommons.org/licenses/by/4.0/). 